\title{
Minimum degree conditions for $H$-linked graphs
}

\author{
Alexandr Kostochka ${ }^{a, b, 1}$, Gexin $\mathrm{Yu}^{\mathrm{a}}$ \\ ${ }^{a}$ Department of Mathematics, University of Illinois, Urbana, IL 61801, USA \\ ${ }^{\mathrm{b}}$ Institute of Mathematics, Novosibirsk 630090, Russia
}

Received 31 March 2004; received in revised form 8 November 2005; accepted 2 November 2006

Available online 23 August 2007

\begin{abstract}
For a fixed multigraph $H$ with vertices $w_{1}, \ldots, w_{m}$, a graph $G$ is $H$-linked if for every choice of vertices $v_{1}, \ldots, v_{m}$ in $G$, there exists a subdivision of $H$ in $G$ such that $v_{i}$ is the branch vertex representing $w_{i}$ (for all $i$ ). This generalizes the notions of $k$-linked, $k$-connected, and $k$-ordered graphs.

Given a connected multigraph $H$ with $k$ edges and minimum degree at least two and $n \geqslant 7.5 k$, we determine the least integer $d$ such that every $n$-vertex simple graph with minimum degree at least $d$ is $H$-linked. This value $D(H, n)$ appears to equal the least integer $d^{\prime}$ such that every $n$-vertex graph with minimum degree at least $d^{\prime}$ is $b(H)$-connected, where $b(H)$ is the maximum number of edges in a bipartite subgraph of $H$.
\end{abstract}

(C) 2007 Elsevier B.V. All rights reserved.

Keywords: Extremal graph problems; Degree conditions; $H$-linked graphs

\section{Introduction}

Let $H$ be a multigraph. An $H$-subdivision in a graph $G$ is a pair of mappings $f: V(H) \rightarrow V(G)$ and $g: E(H)$ into the set of paths in $G$ such that:

(a) $f(u) \neq f(v)$ for all distinct $u, v \in V(H)$ and

(b) for every $u v \in E(H), g(u v)$ is an $f(u) f(v)$-path in $G$, and distinct edges map into internally disjoint paths in $G$.

A graph $G$ is $H$-linked if every injective mapping $f: V(H) \rightarrow V(G)$ can be extended to an $H$-subdivision in $G$. This is a natural generalization of $k$-linkage.

Recall that a graph is $k$-linked if for every list of $2 k$ vertices $\left\{s_{1}, \ldots, s_{k}, t_{1}, \ldots, t_{k}\right\}$, there exist internally disjoint paths $P_{1}, \ldots, P_{k}$ such that each $P_{i}$ is an $s_{i}, t_{i}$-path. By the definition, a graph $G$ is $k$-linked if and only if $G$ is $H$-linked for every graph $H$ with $|E(H)|=k$ and $\delta(H) \geqslant 1$. It is known that a graph $G$ on at least $2 k$ vertices is $k$-linked if and only if $G$ is $M_{k}$-linked, where $M_{k}$ is the matching with $k$ edges.

Let $B_{k}$ denote the (multi)graph with two vertices and $k$ parallel edges. By Menger's theorem, a simple graph $G$ on at least $k+1$ vertices is $k$-connected if and only if $G$ is $B_{k}$-linked.

\footnotetext{
This work was supported by the NSF grants DMS-0099608 and DMS-0400498.

${ }^{1}$ Research was also partially supported by Grants 99-01-00581 and 00-01-00916 of the Russian Foundation for Basic Research.

E-mail address: kostochk@math.uiuc.edu (A. Kostochka).
} 
A graph is $k$-ordered, if for every ordered sequence of $k$ vertices, there is a cycle that encounters the vertices of the sequence in the given order. Let $C_{k}$ denote the cycle of length $k$. Clearly, a simple graph $G$ is $k$-ordered if and only if $G$ is $C_{k}$-linked.

Thus, the notion of $H$-linked graphs is a joint generalization of the notions of $k$-linked, $k$-ordered and $k$-connected graphs. Minimum degree conditions for graphs to be $k$-ordered or $k$-linked were considered by several authors (see $[2,4-10])$. Let $D(n, k)$ be the minimum positive integer $d$ such that every $n$-vertex simple graph with minimum degree at least $d$ is $k$-linked (i.e., $G$ is $H$-linked for every $H$ with $k$ edges). It was proved in [5] that

$$
D(n, k)= \begin{cases}\left\lfloor\frac{n-1,}{n+5 k}-1,\right. & 3 k \leqslant n \leqslant 4 k-2, \\ \left\lceil\frac{n-3}{2}\right\rceil+k, & n \geqslant 4 k-1 .\end{cases}
$$

In fact, Egawa et al. [1] obtained a very similar result earlier in a bit different setting. In [8], we proved that the degree condition can be weakened if $H$ has minimum degree at least two.

Theorem 1. Let $H$ be a loopless graph with $k$ edges and $\delta(H) \geqslant 2$. Every simple graph $G$ of order $n \geqslant 5 k+6$ with $\delta(G) \geqslant\lceil(n+k) / 2\rceil-1$ is H-linked.

The minimum degree condition in Theorem 1 is sharp for all bipartite graphs $H$. The restriction $n \geqslant 5 k+6$ probably can be weakened to about $n \geqslant 3 k$, but not more. The main result of the present paper refines the bound of Theorem 1 for non-bipartite connected multigraphs $H$, but under stronger restrictions on $n$.

Theorem 2. Let $H$ be a loopless connected graph with $k$ edges and $\delta(H) \geqslant 2$. Let $b(H)$ denote the maximum number of edges over all bipartite subgraphs of $H$. Then every simple graph $G$ of order $n \geqslant 7.5 k$ with $\delta(G) \geqslant\lceil(n+b(H)) / 2\rceil-1$ is H-linked.

In the next section we present examples illustrating the theorem and start the proof of the upper bound. We assume that there is no appropriate $H$-subdivision for some choice of branching vertices in $G$ and consider an optimal in some sense subgraph with a vertex set $X$. In Section 3, we estimate $|X|$. In Section 4 we finish the proof.

\section{Preliminaries}

First, we observe that the restriction $\delta(G) \geqslant\lceil(n+b(H)) / 2\rceil-1$ in Theorem 2 cannot be weakened for any $n \geqslant 3 k$ and any $H$. Indeed, let $G$ be the $n$-vertex graph with $V(G)=V_{0} \cup V_{1} \cup V_{2}$ such that $G\left[V_{1}\right]=K_{\lceil(n-b(H)+1) / 2\rceil}$, $G\left[V_{2}\right]=K_{\lfloor(n-b(H)+1) / 2\rfloor}$, and each vertex in $V_{0}$ (with $\left.\left|V_{0}\right|=b(H)-1\right)$ is adjacent to all other vertices in $G$. Then $\delta(G)=\lfloor(n+b(H)-1) / 2\rfloor-1$.

Suppose that $b(H)$ edges in $H$ connect disjoint $X \subset V(H)$ and $Y=V(H)-X$. We claim that $G$ does not contain a subdivision of $H$ such that $X$ is mapped into $V_{1}$ and $Y$ is mapped into $V_{2}$. This is because $b(H)$ edges of $H$ should be mapped into $b(H)$ internally disjoint $V_{1}, V_{2}$-paths passing through $V_{0}$, but $\left|V_{0}\right|=b(H)-1$.

Now we start the proof of the upper bound. Let $f: V(H) \rightarrow V(G)$ be an injective mapping and $W=f(V(H))$. Let $E(H)=\left\{e_{j}=u_{j}^{0} v_{j}^{0}: 1 \leqslant j \leqslant k\right\}$. Let $u_{j}=f\left(u_{j}^{0}\right)$ and $v_{j}=f\left(v_{j}^{0}\right)$. Since $\delta(H) \geqslant 2$, we have $|W|=|V(H)| \leqslant k$.

Say that a family $\mathscr{C}$ of the form $\left\{P_{1}, \ldots, P_{k}\right\}$ is a partial $H$-linkage if each $P_{j}$ is either the set $\left\{u_{j}, v_{j}\right\}$ or a $u_{j}, v_{j}$-path and the following properties hold:

(1) $|X| \leqslant|W|+2(k-b(H)+\alpha)+3$, where $X=\bigcup_{j=1}^{k} V\left(P_{j}\right)$ and $\alpha$ is the number of $P_{j}$ 's that are paths and

(2) the internal vertices of the paths $P_{j}$ 's are pairwise disjoint and disjoint from $W$.

Consider $\mathscr{C}_{0}=\left\{\left\{u_{1}, v_{1}\right\}, \ldots,\left\{u_{k}, v_{k}\right\}\right\}$. This family satisfies properties (1) and (2) above with $X=\bigcup_{j=1}^{k}\left\{u_{j}, v_{j}\right\}=W$ and $\alpha=0$. Therefore, $\mathscr{C}_{0}$ is a partial $H$-linkage. If all the $P_{j}$ 's in a partial $H$-linkage $\mathscr{C}$ are paths, then $\mathscr{C}$ is an $H$-subdivision in $G$. 
A partial $H$-linkage $\mathscr{C}=\left\{P_{1}, \ldots, P_{k}\right\}$ is optimal, if as many as possible of the $P_{j}$ 's are paths and subject to this the set $X=\bigcup_{j=1}^{k} V\left(P_{j}\right)$ is as small as possible. We will prove that each optimal $H$-linkage is an $H$-subdivision in $G$.

Suppose for a contradiction that $\mathscr{C}=\left\{P_{1}, \ldots, P_{k}\right\}$ is an optimal partial $H$-linkage but is not an $H$-subdivision. Let, for definiteness, $P_{k}=\left\{u_{k}, v_{k}\right\}$ and $u_{k} v_{k} \notin E(G)$. Let $X=\bigcup_{j=1}^{k} V\left(P_{j}\right), x=u_{k}$, and $y=v_{k}$. Let $A=N(x)-X, B=N(y)-X$, and $R=V(G)-(X \cup A \cup B)$.

It is well known (see, e.g., [11]) that

$$
b(H) \geqslant(k+1) / 2
$$

for every $H$ with $k>0$ edges. Therefore, each of $A$ and $B$ has size at least

$$
\begin{aligned}
\delta(G)-(|X|-2) & \geqslant \frac{n+b(H)-2}{2}-(|W|+2(k-b(H)+(k-1))+3-2) \\
& \geqslant \frac{7.5 k+b(H)-2}{2}-5 k+2 b(H)+1=1.25(2 b(H)-k)>1.25 .
\end{aligned}
$$

It follows that we may choose distinct $a_{1}, a_{2} \in A$ and $b_{1}, b_{2} \in B$.

For $v \in V(G)$, let $d_{j}(v)$ denote the number of neighbors of $v$ 'inside' $P_{j}$ plus $\beta_{j}=1 / \operatorname{deg}_{H}\left(u_{j}^{0}\right)$ if $u_{j} \in N_{G}(v)$ and plus $\gamma_{j}=1 / \operatorname{deg}_{H}\left(v_{j}^{0}\right)$ if $v_{j} \in N_{G}(v)$. For example, if $P_{j}=u_{j} w_{1} w_{2} v_{j}, \operatorname{deg}_{H}\left(u_{j}^{0}\right)=3$ and $v$ is adjacent to $u_{j}$ and $w_{2}$ in $P_{j}$, then $d_{j}(v)=\frac{4}{3}$. It is easy to check that

$$
\sum_{j=1}^{k} d_{j}(v)=\left|N_{G}(v) \cap X\right| \quad \forall v \in V(G) .
$$

Let $l_{p}$ be the number of $P_{j}$ 's of length $p$ for $p \geqslant 1$, and $l_{0}$ be the number of $P_{j}$ that are not paths. Then

$$
|X|=|W|+\sum_{p \geqslant 1}(p-1) l_{p}=\sum_{j=1}^{k}\left(\beta_{j}+\gamma_{j}\right)+\sum_{p \geqslant 1}(p-1) l_{p}
$$

and

$$
k=\sum_{p \geqslant 0} l_{p}=\alpha+l_{0}
$$

\section{A bound on the size of $X$}

We will assume that every path $P_{j}$ is of the form $P_{j}=u_{j}, w_{1, j}, \ldots, w_{p_{j}-1, j}, v_{j}$. Sometimes, for simplicity we will write $p$ instead of $p_{j}$ and $w_{i}$ instead of $w_{i, j}$ if $j$ is clear from the context. In the rest of the paper, for every $j=1, \ldots, k$, we denote $\beta_{j}=1 / \operatorname{deg}_{H}\left(u_{j}^{0}\right), \gamma_{j}=1 / \operatorname{deg}_{H}\left(v_{j}^{0}\right), M_{j}=d_{j}(x)+d_{j}(y)$, and $L_{j}=d_{j}\left(a_{1}\right)+d_{j}\left(a_{2}\right)+d_{j}\left(b_{1}\right)+d_{j}\left(b_{2}\right)$.

The following lemma (which is Lemma 5 in [8]) will be very helpful.

Lemma 3. For a $P_{j}=u_{j}, w_{1}, \ldots, w_{p-1}, v_{j}$, let $s_{j}=M_{j}+0.5 L_{j}, \beta=\beta_{j}$, and $\gamma=\gamma_{j}$. Define

$$
D_{1}(p, \beta, \gamma)= \begin{cases}p+1+2 \beta+2 \gamma, & \text { for } p \leqslant 1, \\ p+3+2 \beta+2 \gamma, & \text { for } p \geqslant 2 .\end{cases}
$$

Then

(a) $s_{j} \leqslant D_{1}(p, \beta, \gamma)$ and

(b) $s_{k} \leqslant 2\left(\beta_{k}+\gamma_{k}\right)$.

Furthermore, if $x y \notin E(G)$, then $s_{k}=\beta_{k}+\gamma_{k}$.

Based on Lemma 3, we prove the following. 
Lemma 4. Let $Z=\left\{a_{1}, a_{2}, b_{1}, b_{2}\right\}$ and $V_{0}=(A \cup B)-Z-N_{G}(Z)$. Then $|X| \leqslant|W|+2(\alpha+k-b(H))-|R|-\left|V_{0}\right|$.

Proof. Let

$$
\Sigma^{\prime}=\operatorname{deg}_{G}(x)+\operatorname{deg}_{G}(y)+\frac{1}{2}\left(\operatorname{deg}_{G}\left(a_{1}\right)+\operatorname{deg}_{G}\left(a_{2}\right)+\operatorname{deg}_{G}\left(b_{1}\right)+\operatorname{deg}_{G}\left(b_{2}\right)\right) .
$$

Every vertex $w \in A \cup B$ contributes to $\Sigma^{\prime}$ at most 2: if $w \in A$ (respectively, $w \in B$ ), then it is not adjacent to $y, b_{1}$, and $b_{2}$ (respectively, to $x, a_{1}$, and $a_{2}$ ). By the definition, every vertex in $V_{0}$ is not adjacent to any vertex in $Z$ and to at least one of $x$ and $y$. Therefore, every vertex in $V_{0}$ contributes to $\Sigma^{\prime}$ at most 1 . Furthermore, every $z \in Z$ contributes to $\Sigma^{\prime}$ at most 1.5 , since it is not adjacent to itself. Thus, in total $A \cup B$ contributes to $\Sigma^{\prime}$ at most $2|A \cup B|-\left|V_{0}\right|-0.5|Z|$. Every $r \in R$ contributes to $\Sigma^{\prime}$ at most 2. By the definition, for every $j$, the vertices of $P_{j}$ contribute to $\Sigma^{\prime}$ exactly $s_{j}$. Therefore,

$$
\Sigma^{\prime} \leqslant 2|A \cup B|-2+2|R|+\sum_{j=1}^{k} s_{j}-\left|V_{0}\right| .
$$

By Lemma 3,

$$
\begin{aligned}
\sum_{j=1}^{k} s_{j} & \leqslant l_{0}+2 l_{1}+\sum_{p \geqslant 2}(p+3) l_{p}+2 \sum_{j=1}^{k}\left(\beta_{j}+\gamma_{j}\right)-1 \\
& =l_{0}+2 l_{1}+\sum_{p \geqslant 2}(p+3) l_{p}+2|W|-1 .
\end{aligned}
$$

Therefore,

$$
\Sigma^{\prime} \leqslant 2(|A|+|B|+|W|)+2|R|-\left|V_{0}\right|-3+l_{0}+2 l_{1}+\sum_{p \geqslant 2}(p+3) l_{p} .
$$

Combining with (4) and (5), we get

$$
|X|+\Sigma^{\prime} \leqslant 2 n+|W|+2 k+2 \alpha-3-l_{0}-2 l_{1}-\left|V_{0}\right| .
$$

By $(2), \delta(G) \geqslant((n+b(H)) / 2)-1$ and hence $\Sigma^{\prime} \geqslant 2 n+2 b(H)-4$. Thus,

$$
|X| \leqslant|W|+2(k-b(H)+\alpha)-l_{0}-2 l_{1}-\left|V_{0}\right|+1 \leqslant|W|+2(k-b(H)+\alpha)-\left|V_{0}\right| .
$$

If an $r \in R$ has a neighbor $a_{0} \in A$ and a neighbor $b_{0} \in B$, then one can add to $\mathscr{C}$ the path $P_{k}=x, a_{0}, r, b_{0}, y$. The new set of paths will be a better partial linkage, since the new $X$ would have size at most $|W|+2(k-b(H)+\alpha)+$ $3=|W|+2(k-b(H)+\alpha+1)+1$. Since this contradicts the choice of $\mathscr{C}$, no $r \in R$ has both a neighbor in $A$ and a neighbor in $B$. Thus, every $r \in R$ contributes to $\Sigma^{\prime}$ at most 1 , and (7) becomes

$$
\Sigma^{\prime} \leqslant 4 \cdot 1.5+2(|A \cup B|-4)+|R|+\sum_{j=1}^{k} s_{j}-\left|V_{0}\right| .
$$

Correspondingly, (10) transforms into

$$
|X| \leqslant|W|+2(k-b(H)+\alpha)-\left|V_{0}\right|-|R| .
$$

This lemma has the following two immediate consequences.

Lemma 5. $|A|+|B|>3 k$.

Proof. By Lemma 4, $|A|+|B|=n-(|X|+|R|) \geqslant n-(|W|+2(k-b(H)+\alpha)) \geqslant 7.5 k-(k+2(k-((k+1) / 2)+$ $k-1))>3 k$. 
Lemma 6. Each $v \in V(G)$ is adjacent to at least three vertices in $A \cup B-V_{0}$. In particular, either $v$ has two neighbors in $A$ that belong or are adjacent to the set $\left\{a_{1}, a_{2}\right\}$, or two neighbors in $B$ that belong or are adjacent to the set $\left\{b_{1}, b_{2}\right\}$.

Proof. By Lemma 4, $\delta(G)-\left(|X|+|R|+\left|V_{0}\right|\right) \geqslant 0.5(7.5 k+b(H)-2)-|W|-2(k-b(H)+\alpha) \geqslant 3.75 k+0.5 b(H)-$ $1-k-2(k-b(H)+k-1)=1.25(2 b(H)-k)+1>2$. Thus each vertex has at least three neighbors in $V(G)-X-$ $R-V_{0}$.

For given $a_{1}, a_{2} \in A, b_{1}, b_{2} \in B$, let $A^{\prime \prime}=A^{\prime \prime}\left(a_{1}, a_{2}\right)$ (respectively, $\left.B^{\prime \prime}=B^{\prime \prime}\left(b_{1}, b_{2}\right)\right)$ denote the set of vertices in $X$ having at least two neighbors in $A$ (respectively, in $B$ ) that belong or are adjacent to the set $\left\{a_{1}, a_{2}\right\}$ (respectively, $\left.\left\{b_{1}, b_{2}\right\}\right)$. The above lemma yields that for every choice of $a_{1}, a_{2}, b_{1}$, and $b_{2}$,

$$
A^{\prime \prime} \cup B^{\prime \prime}=X \text {. }
$$

\section{Proof of Theorem 2}

Lemma 7. For every non-adjacent $s, t \in A($ or $B),|N(s) \cap N(t)-X| \geqslant 3$.

Proof. Suppose to the contrary that $a_{1}, a_{2} \in A, a_{1} a_{2} \notin E(G)$ and the cardinality of the set $T$ of common neighbors of $a_{1}$ and $a_{2}$ outside of $X$ is at most two. Consider arbitrary $b_{1}, b_{2} \in B$ and let $Z=\left\{a_{1}, a_{2}, b_{1}, b_{2}\right\}$. Then the contribution of every $a \in A-Z-T$ to the sum $\Sigma^{\prime}$ defined in (6) is at most 1.5. Thus, repeating the proof of Lemma 4 , instead of (11), we will get $|X| \leqslant|W|-|R|+2(k-b(H)+\alpha)-\left|V_{0}\right|-0.5\left(\left|A-V_{0}\right|-4\right)$. In other words,

$$
|X|+0.5|A|+|R| \leqslant|W|+2(k-b(H)+\alpha)+2 \leqslant 5 k-2 b(H) .
$$

On the other hand, $\operatorname{deg}_{G-X}\left(a_{1}\right)+\operatorname{deg}_{G-X}\left(a_{2}\right) \leqslant|A|+|T|+|R|-2$ (the -2 arises because neither of $a_{1}$ and $a_{2}$ is adjacent to $a_{1}$ or $a_{2}$ ). It follows that

$$
2 \frac{n+b(H)}{2}-2 \leqslant 2 \delta(G) \leqslant 2|X|+|A|+|R|,
$$

which together with (13) yields $n+b(H)-2 \leqslant 2(5 k-2 b(H))$. Thus, $n \leqslant 10 k-5 b(H)+2 \leqslant 10 k-5((k+1) / 2)+$ $2=7.5 k-0.5$, a contradiction.

For the rest of the section, we fix some distinct $a_{1}, a_{2} \in A$ and $b_{1}, b_{2} \in B$, and let $A^{\prime \prime}=A^{\prime \prime}\left(a_{1}, a_{2}\right)$ and $B^{\prime \prime}=B^{\prime \prime}\left(b_{1}, b_{2}\right)$. The next fact from [6] was used in [8].

Lemma 8. Let $X$ be optimal, $1 \leqslant j \leqslant k-1$, and either $\left\{u_{j}, v_{j}\right\} \subset A^{\prime \prime}$ or $\left\{u_{j}, v_{j}\right\} \subset B^{\prime \prime}$. Then for each a $\in A$ and $b \in B$,

$$
\left(N(a) \cap N(b) \cap P_{j}\right) \backslash\left\{u_{j}, v_{j}\right\}=\emptyset .
$$

Proof. Assume to the contrary that $r \in N(a) \cap N(b) \cap P_{j} \backslash\left\{u_{j}, v_{j}\right\}$. Let $P_{k}^{\prime}=(x, a, r, b, y)$. Without loss of generality, assume that $\left\{u_{j}, v_{j}\right\} \subset A^{\prime \prime}$. Then there exist $s \in N\left(u_{j}\right) \cap A \backslash\{a\}$ and $t \in N\left(v_{j}\right) \cap A \backslash\{a\}$. If $s=t$ or $s$ is adjacent to $t$, then let $P_{j}^{\prime}=\left(u_{j}, s, t, v_{j}\right)$.

If $s$ and $t$ are non-adjacent, then by Lemma 7, we have $|(N(s) \cap N(t)) \backslash X| \geqslant 3$, and therefore there exists $q \in N(s) \cap N(t) \backslash(X \cup\{a, b\})$. In this case, let $P_{j}^{\prime}=\left(u_{j}, s, q, t, v_{j}\right)$. In both cases, $P_{j}^{\prime}$ is a path disjoint from $P_{k}^{\prime}$. Thus, in both cases we increase the number of $P_{j}$ 's that are paths by one and, by (11), maintain $|X| \leqslant|W|+2(k-b(H)+\alpha+1)+2$. This is a contradiction.

Lemma 9. Let $X$ be optimal, $1 \leqslant j \leqslant k-1, P_{j}=\left(w_{0}, w_{1}, \ldots, w_{p}\right)$, where $w_{0}=u_{j} \in A^{\prime \prime}$ and $w_{p}=v_{j} \in B^{\prime \prime}$. If some $w_{i}, 1 \leqslant i \leqslant p-1$ has a neighbor $a_{0} \in A \cup\{x\}$ and a neighbor $b_{0} \in B \cup\{y\}$, then each $w_{i^{\prime}}$ for $i<i^{\prime} \leqslant p$ has no neighbors in $A-a_{0}$ and each $w_{i^{\prime \prime}}$ for $0 \leqslant i^{\prime \prime}<i$ has no neighbors in $B-b_{0}$.

Proof. Suppose some $w_{i^{\prime}}$ for $i<i^{\prime} \leqslant p$ has a neighbor $a^{\prime} \in A-a_{0}$. By the definition of $A^{\prime \prime}, u_{j}$ has a neighbor $a^{\prime \prime} \in A-a_{0}$. By Lemma 7, the length of a shortest path $P^{\prime}$ from $a^{\prime \prime}$ to $a^{\prime}$ in $G\left[A-a_{0}\right]$ is at most two. Thus, we 
can replace $P_{j}$ by the path $\left(u_{j}, a^{\prime \prime}, P^{\prime}, a^{\prime}, w_{i^{\prime}}, P_{j}^{\prime}, v_{j}\right)$ (where $P_{j}^{\prime}$ is the part of $P_{j}$ connecting $w_{i^{\prime}}$ with $\left.v_{j}\right)$ and add the path $P_{k}=\left(x, a_{0}, w_{i}, b_{0}, y\right)$. The new set of $\alpha+1$ paths has at most $|X|+5$ vertices, which by (11) is at most $|W|+2(k-b(H)+\alpha+1)+3$, a contradiction to the choice of $\mathscr{C}$.

Similarly to $d_{j}(v)$, let $d_{j}(u, v)$ denote the number of common neighbors of $u$ and $v$ 'inside' $P_{j}$ plus $\beta_{j} \cdot \mid N(u) \cap$ $N(v) \cap\left\{u_{j}\right\} \mid$ plus $\gamma_{j} \cdot\left|N(u) \cap N(v) \cap\left\{v_{j}\right\}\right|$.

Lemma 10. Let $\mathscr{C}$ be optimal, $a \in A, b \in B$. Then there exists some $j=j(a, b)$ such that $d_{j}(a, b)>1$.

Proof. Since $N(a) \cap N(b) \cap(V(G)-X+x+y)=\emptyset$, we have

$$
\sum_{j=1}^{k-1} d_{j}(a, b)=|N(a) \cap N(b)| \geqslant 2 \delta(G)-(n-2) \geqslant b(H) .
$$

Suppose that $d_{j}(a, b) \leqslant 1$ for each $1 \leqslant j \leqslant k-1$. We will find an edge cut in $H$ with more than $\sum_{j=1}^{k-1} d_{j}(a, b)$ edges, a contradiction to (14). Let $E^{\prime}$ be the set of edges $e_{j}$ in $H$ such that an internal vertex of $P_{j}$ is in $N(a) \cap N(b)$. Let $V^{\prime}$ be the set of vertices $u^{0}$ in $H$ such that the vertex $f\left(u^{0}\right)$ (i.e., the branching vertex in $G$ corresponding to $u^{0}$ ) is in $N(a) \cap N(b)$. By our assumption, no vertex in $V^{\prime}$ is incident to an edge in $E^{\prime}$, and for each $e_{j} \in E^{\prime}$, the path $P_{j}$ contains exactly one vertex of $N(a) \cap N(b)$. Thus, it is enough to find in $H$ an edge cut of size greater than $\left|E^{\prime}\right|+\left|V^{\prime}\right|$.

By Lemma 8, for each $e_{j} \in E^{\prime}$, either $u_{j} \in A^{\prime \prime}-B^{\prime \prime}$ and $v_{j} \in B^{\prime \prime}-A^{\prime \prime}$ or $v_{j} \in A^{\prime \prime}-B^{\prime \prime}$ and $u_{j} \in B^{\prime \prime}-A^{\prime \prime}$. Recall that $x=f\left(u_{k}^{0}\right), y=f\left(v_{k}^{0}\right), x \in A^{\prime \prime}-B^{\prime \prime}$ and $y \in B^{\prime \prime}-A^{\prime \prime}$. It follows that the set $E^{\prime} \cup\left\{e_{k}\right\}$ is contained in an edge-cut in $H$. Let $V_{1}$ and $V_{2}$ be the disjoint subsets of $V(H)$ such that:

(a) each edge in $E^{\prime} \cup\left\{e_{k}\right\}$ is incident to a vertex in $V_{1}$ and a vertex in $V_{2}$ and

(b) each vertex in $V_{1} \cup V_{2}$ is incident to an edge in $E^{\prime} \cup\left\{e_{k}\right\}$.

By the above, $V^{\prime} \cap\left(V_{1} \cup V_{2}\right)=\emptyset$ and hence $\left|V(H)-\left(V_{1} \cup V_{2}\right)\right| \geqslant\left|V^{\prime}\right|$. Since $H$ is connected, there is a vertex $u^{0}$ adjacent to $V_{1} \cup V_{2}$. If $u^{0}$ is adjacent to $V_{1}$, then we add $u^{0}$ to $V_{2}$, otherwise add it to $V_{1}$. In any case the number of edges between the new $V_{1}$ and $V_{2}$ is greater than between the old ones. We continue adding vertices to $V_{1} \cup V_{2}$ so that with each added vertex, the number of edges between $V_{1}$ and $V_{2}$ grows by at least one. When we add the last vertex of $H$, we get a partition $\left(V_{1}, V_{2}\right)$ of $V(H)$ such that the number of edges between $V_{1}$ and $V_{2}$ is at least

$$
\left|E^{\prime} \cup\left\{e_{k}\right\}\right|+\left|V(H)-\left(V_{1} \cup V_{2}\right)\right| \geqslant\left|E^{\prime}\right|+1+\left|V^{\prime}\right|,
$$

a contradiction to (14).

Lemma 11. Let $X$ be optimal, $1 \leqslant j \leqslant k-1$. Then there is at most one $a \in A$, such that there is more than one $b \in B$ with $j=j(a, b)$.

Proof. Let $P_{j}=\left(w_{0}, w_{1}, \ldots, w_{p}\right)$, where $w_{0}=u_{j}$ and $w_{p}=v_{j}$. Assume to the contrary that there are $a_{1}, a_{2} \in A$ and $b_{1}, b_{2}, b_{3}, b_{4} \in B$ such that $j\left(a_{1}, b_{1}\right)=j\left(a_{1}, b_{2}\right)=j\left(a_{2}, b_{3}\right)=j\left(a_{2}, b_{4}\right)=j$, where $a_{1} \neq a_{2}, b_{1} \neq b_{2}, b_{3} \neq b_{4}$. By Lemma 8, we may assume that $u_{j} \in A^{\prime \prime} \backslash B^{\prime \prime}$ and $v_{j} \in B^{\prime \prime} \backslash A^{\prime \prime}$.

Since $\beta_{j}+\gamma_{j} \leqslant 1$, there exists $i, 1 \leqslant i \leqslant p-1$, such that $w_{i} \in N\left(a_{1}\right) \cap N\left(b_{1}\right)$. Since $b_{3} \neq b_{4}$, we may assume that $b_{3} \neq b_{1}$. By Lemma 9, no vertex in $V\left(P_{j}\right)-w_{i}$ can belong to $N\left(a_{2}\right) \cap N\left(b_{3}\right)$. This contradicts the fact that $d_{j}\left(a_{2}, b_{3}\right)>1$.

By Lemma $5,|A|+|B|>2 k$. We may assume that $|A| \leqslant|B|$. Thus, $|B| \geqslant k$. If $|A| \geqslant k$, then since $|B| \geqslant k$, by Lemma 10, for each $a \in A$ there is some $j(a)$ and $b_{1}(a)$ and $b_{2}(a)$ such that $j(a)=j\left(a, b_{1}(a)\right)=j\left(a, b_{2}(a)\right)$. Furthermore, since $|A| \geqslant k$, for some $a_{1}, a_{2} \in A$, the indices $j\left(a_{1}\right)$ and $j\left(a_{2}\right)$ are the same. This contradicts Lemma 11.

Thus, we may assume that $|A|<k$. Since $|B| \geqslant k$, for each $a \in A$ there is some $j(a)$ and $b_{1}(a)$ and $b_{2}(a)$ such that $j(a)=j\left(a, b_{1}(a)\right)=j\left(a, b_{2}(a)\right)$. Let $J=\{j(a) \mid a \in A\}$. By Lemma 11, the indices $j(a)$ are distinct for distinct $a \in A$ and hence $|J|=|A|$.

Lemma 12. Suppose that $j \in J$. Then $x$ is not adjacent to some interior vertex of $P_{j}$. 
Proof. Let $P_{j}=\left(w_{0}, w_{1}, \ldots, w_{p}\right)$, where $w_{0}=u_{j}$ and $w_{p}=v_{j}$. By the definition of $J$, there exists $a \in A$ and $b_{1}, b_{2} \in B$ such that $d_{j}\left(a, b_{1}\right)$ and $d_{j}\left(a, b_{2}\right)>1$. Since $\beta_{j}+\gamma_{j} \leqslant 1$, this implies that $p \geqslant 2$. Assume that $u_{j} \in A^{\prime \prime}-B^{\prime \prime}$ and $v_{j} \in B^{\prime \prime}-A^{\prime \prime}$.

Since $u_{j} \notin B^{\prime \prime}$, we may assume that $u_{j} b_{1} \notin E(G)$. Let $w_{i^{\prime}}, w_{i^{\prime \prime}} \in N(a) \cap N\left(b_{1}\right)$ and $i^{\prime}<i^{\prime \prime}$. By the choice, $1 \leqslant i^{\prime} \leqslant p-1$. If $x w_{i^{\prime}} \in E(G)$, then we get a contradiction to Lemma 9 with $a_{0}=x$, since $w_{i^{\prime \prime}} a \in E(G)$. Thus, $x w_{i^{\prime \prime}} \notin E(G)$.

Proof (End of the proof). By Lemma 12, $x$ is not adjacent to at least $|J|$ vertices in $X-W$. It also is not adjacent to itself. Thus, $|N(x) \cap X| \leqslant|X|-|J|-1 \leqslant|W|+2(k-b(H)+k-1)-|J|-1 \leqslant 5 k-2 b(H)-3-|J|$. Since $|J|=|A|=|N(x)-X|$, we get

$$
\frac{n+b(H)}{2}-1 \leqslant \operatorname{deg}(x) \leqslant 5 k-2 b(H)-3,
$$

which yields $n \leqslant 10 k-5 b(H)-1<7.5 k-2$, a contradiction.

\section{Acknowledgment}

We thank the referees for their helpful comments.

\section{References}

[1] Y. Egawa, R.J. Faudree, E. Györi, Y. Ishigami, R.H. Schelp, H. Wang, Vertex-disjoint cycles containing specified edges, Graphs Combin. 16 (2000) 81-92.

[2] J. Faudree, R. Faudree, R. Gould, M. Jacobson, L. Lesniak, On k-ordered graphs, J. Graph Theory 35 (2000) 69-82.

[4] R.J. Gould, Advances on the Hamiltonian problem—a survey, Graphs Combin. 19 (2003) 7-52.

[5] K. Kawarabayashi, A. Kostochka, G. Yu, On sufficient degree conditions for a graph to be k-linked, Combin. Probab. Comput. 15 (2006) 685-694.

[6] H.A. Kierstead, G. Sárközy, S. Selkow, On k-ordered hamiltonian graphs, J. Graph Theory 32 (1999) 17-25.

[7] A. Kostochka, G. Yu, On $H$-linked graphs, Oberwolfach Rep. 1 (2004) 42-45.

[8] A. Kostochka, G. Yu, An extremal problem for $H$-linked graphs, J. Graph Theory 50 (2005) 321-339.

[9] L. Ng, M. Schultz, $k$-Ordered hamiltonian graphs, J. Graph Theory 2 (1997) 45-57.

[10] R. Thomas, P. Wollan, An improved linear edge bound for graph linkage, European J. Combin. 26 (2005) 309-324.

[11] D.B. West, Introduction to Graph Theory, second ed., Prentice-Hall, Upper Saddle River, 2001. 